\title{
Development of Speech Commentary Software about Inquiry of Ship Engine Damage Cases
}

\author{
Jiang Lu ${ }^{1 *}$, Liu Jiang ${ }^{2}$ and $\mathrm{HU}$ Yi-huai $^{2}$ \\ ${ }^{1}$ Shanghai Maritime Academy, Shanghai, Pudong new area, 200120, China \\ ${ }^{2}$ Shanghai Maritime University, Shanghai, Pudong new area, 201306, China
}

\begin{abstract}
On the basis of the analysis about the Chinese-English engine of Microsoft Speech SDK, the paper achieves the development of speech commentary software about inquiry of ship engine damage cases with Visual Basic. And it also introduces carefully the development thoughts, programming ways and application cases of this software.
\end{abstract}

\section{Achievement thoughts}

Speech Recognition (SR) and Text to Speech (TTS) have always been the hotspot of computer application. If you want to develop the application programs with functions of Speech Recognition and Text to Speech on the platform of Windows, it is a better choice for you to use Microsoft Speech SDK. Microsoft Speech SDK is a toolkit issued by Microsoft, which can support the development of speech application programs. Its latest version is 5.1 and can be downloaded for free on the website of Microsoft.

If you want it to support Chinese, you still should download the relevant Language Pack. After downloading, you should first install Speech SDK, and then install the language pack of simplified Chinese so that your computer has the function of Chinese and English.

The technology of Speech Recognition has been the hotspot concerned at home and abroad because of its practicability and interest. The companies of Microsoft, IBM and so on have issued in succession the products of Speech Recognition on the basis of PC. Among them, Microsoft's Speech SDK completely supports the system development of simplified Chinese speech, and so it is an ideal tool to develop speech software.

It is developed on the basis of the COM standard, and its bottom protocol is completely independent from the layer of application programs with the form of COM components, so it shields the complicated speech technologies for the application programmers, which fully reflects the advantage of COM. Speech Recognition is managed by Recognition Engine, which can achieve the recognition of Chinese from speech. Text to Speech is managed by TTS Engine mentioned above, which can achieve speech reading.

Software programmers only concentrate on their own application, transfer the access (SAPI) of relevant speech application programs to achieve speech functions, and its structure is shown by Figure 1 .

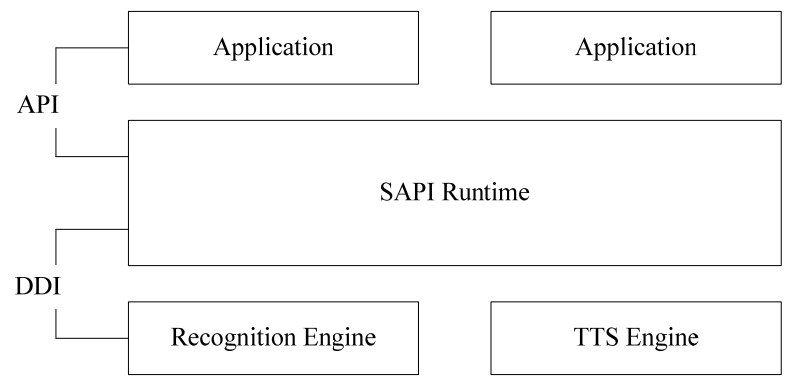

Figure 1. Structure chart of TTS on the basis of Speech SDK

\section{Software Programming}

The category of SpVoice is the core category of TTS supporting. The object of SpVoice transfers TTS engine to achieve the function of reading.

The category of SpVoice has the following main attributes:

Voice: It means pronunciation, which is equivalent to the person who is reading, including Microsoft Mary, Microsoft Mike, Microsoft Sam and Microsoft Simplified Chinese. The first three categories among them only can read English and the last one can read both Chinese and English.

Rate: the speed of reading, ranging from -10 to +10 . The bigger the number is, the quicker the speed is.

Volume: Volume, ranging from 0 to 100 . The bigger the number is, the larger the volume is.

SpVoice has the following main ways:

Speak: It transfers text information to speech and reads according to the designated parameter. This way has two parameters of Text and Flags, which respectively mean the text of reading and the way of reading (synchronous, asynchronous and so on).

Pause: It pauses to use all the reading process of this object. This way has no parameter.

Resume: It resumes the relevant paused reading process of this object. This way has no parameter.

Here are parts of codes.

Definition of the initial variable

Option Explicit

Dim WithEvents Voice As SpVoice

Dim m_speakFlags As SpeechVoiceSpeakFlags 
Const NoAudioOutput $=$ "No audio ouput object available"

Private m_bSpeaking As Boolean

Private m_bPaused As Boolean

'Codes of form initialization

Private Sub Form_Load()

Me. Width $=$ Screen. Width

Me.Height $=$ Screen. Height

Set Voice $=$ New SpVoice

Dim Token As ISpeechObjectToken

For Each Token In Voice.GetVoices Next

VoiceCB.AddItem (Token.GetDescription())

VoiceCB.ListIndex $=0$

RateSldr.Value $=$ Voice. Rate

VolumeSldr. Value $=$ Voice . Volume

m_speakFlags=SVSFlagsAsync Or

SFPurgeBeforeSpeak Or SVSFIsXML

SetSpeakingState False, False

End Sub

'Codes of clicking "Start playing”

Private Sub SpeakBtn_Click()

If rtbFaultProcess. Text = "' Then Exit Sub

End If

If Not (m_bPaused And m_bSpeaking) Then

'Voice.Speak rtbFaultProcess.Text, m_speakFlags Voice.Speak rtbFaultProcess.Text \& "Analysis and processing" \& rtbFaultMethod.Text \&

"Experience and lessons" \& rtbFaultLesson.Text, SVSFlagsAsync

End If

If m_bPaused Then Voice.Resume

SetSpeakingState True, False

Exit Sub

End Sub

'Codes of "Stop playing"

Private Sub StopBtn_Click()

Voice.Speak vbNullString, SVSFPurgeBeforeSpeak

If m_bPaused Then Voice.Resume

SetSpeakingState False, False

Exit Sub

End Sub

\section{An application case}

Through a complete case, the paper introduces how to develop speech application programs with Microsoft Speech SDK in Visival Basic 6.0.

The function of this case application program is that the customer chooses a kind of ship engine damage accident (for example, diesel engines, connecting rods, auxiliary engines and other equipment), and chooses the detailed description of the relevant engine damage accident about this kind of accidents in the relevant figure.
And then this inquiry software will shift to the window shown by Figure 2 .

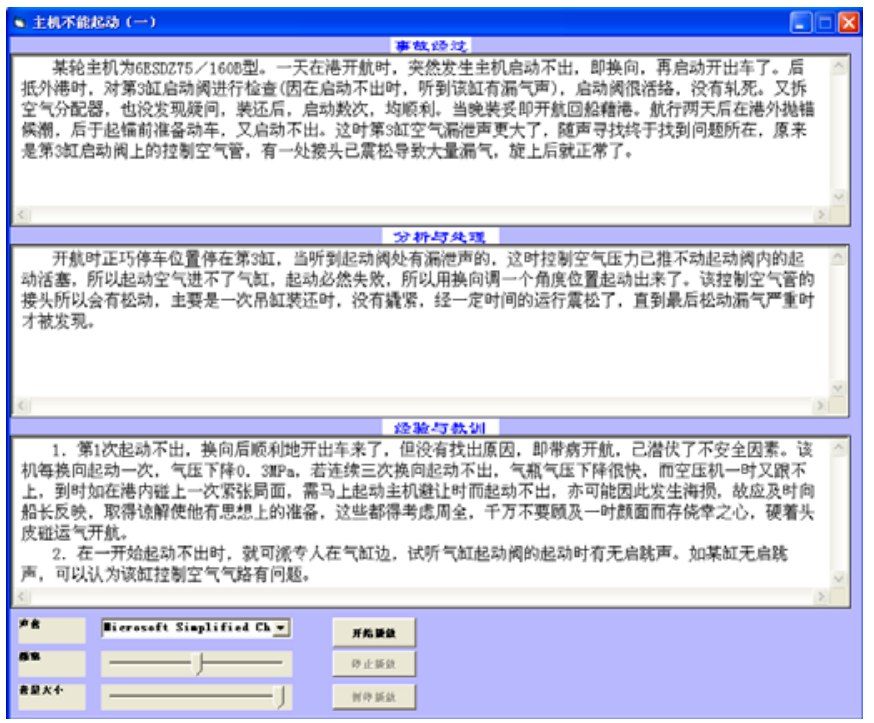

Figure 2. the speech commentary window of the inquiry software about ship engine damage

The contents of Figure 2 are as follows.

\section{The main engine can't be started.}

\begin{tabular}{|l|}
\hline \multicolumn{3}{|c|}{ Accident } \\
\hline The main engine of some ship is the type of \\
6ESDZ75/160B.
\end{tabular}

When it left the port one day, suddenly the main engine could not be started, and then it was reversed and started to leave.

After that, when the ship arrived at the outer port, the starting valve of the third cylinder was checked (because when the main engine could not be started, the crew heard the sound of leak from the cylinder). The starting valve was not stabbed. And then, the air distributor was opened, but there was no problem at all. After being installed, the main engine was started for some times, which was smooth going.

In the same evening, after being installed properly, the ship left to go back to the port of registry.

After a two-day journey, the ship dropped anchor and waited for the tide. When the crew intended to start before weighing anchor, the main engine could not be started.

At this time, the sound of leak from the third cylinder was larger, and then the crew found the problem. In fact, the problem was the controlling air pipe of the starting valve in the third cylinder, where one joint was loose, leading to leak a large amount of air. Then the joint was tightened, and everything went to be normal.

\section{Analysis and processing}


When starting to sail, the third cylinder was at the stoppede position.

Then when the crew heard the sound of leak from the starting valve, controlling air pressure could not push the starting piston in the starting valve, and so the starting air could not go into the cylinder and the start certainly failed, but the main engine could be started when reversed to another angel.

The reason why the joint of the controlling air pipe was loose was that it was not tightened when the cylinder was lifted and installed.

After a period of working time, the joint was loose until it was found to leak air seriously at last.

\section{Experience and lessons}

1. At the first time when the main engine could not started, and then it was reversed to start smoothly, but nobody found the reason, which meant the ship sailed with problems and hid the unsafe factors.

Every time when the main engine was reversed to start, the air pressure reduced $0.3 \mathrm{Mpa}$. If it was reversed for three times but could not be started, the air pressure of the gas cylinder would reduce very quickly, but the air compressor could not follow temporarily.

At that time, if it happened to be in a tense situation and the main engine needed to be started immediately to dodge but could not be started, it would occur to be the average.

So the crew should report to the captain in time to get his forgiveness in order to have ideological preparation. These had to be considered thoroughly.

Don't think about your temporarily prestige and luck to force yourselves to start sailing.

2. When the main engine could not be started at first, it was necessary to send somebody near the cylinder to try to hear whether there was the sound when the starting valve of the cylinder was started.

If there was no sound in some cylinder, this cylinder had the problem in the controlling air pipe.

\begin{tabular}{|ll|}
\hline Voice & start transformation \\
\hline Rate & stop transformation \\
\hline Volume & pause transformation \\
\hline
\end{tabular}

In the window of Figure 2, when we click the right bottom button of "start playing", the computer will automatically play the accident, analysis and processing and experience and lessons of this case. Furthermore, it also can adjust the reading speed and volume according to needs, which is shown by Figure 2 .

\section{Conclusion}

It is the most important to ensure the safety of the ship in navigation. The main work of the marine engineer is to ensure the normal operation of the ship machinery. The damage of the mechanical parts of the ship is an important cause of the ship's navigation accident.

Through further studying and analysis of similar accidents, we can improve the technical service level of the crew and the maintenance staff.

This software has collected more than 400 engine damage cases from the domestic main shipping companies such as China Shipping Group and China Ocean Shipping Company in the last over 30 years.

This software can introduce and analyze ship engine damage cases, and use the speech technology to achieve the live commentary, so we can more vividly understand ship engine damage cases.

The teachers and students of Marine Engineering Major can master the cause, the solution and the experience of different accidents by this software. It is the useful way to combine the theory with practice in the process of teaching and training.

In the course of classroom teaching, rich teaching case can make the boring content more easily understood. Students can also improve their knowledge structure by this software. It has a positive meaning for the students to the working environment more quickly in the first ship navigation.

The crew can study on the ship by using the software. And through checking and analyzing different kinds of accidents and unsafe factors of ships and according to the existing problems and the technical situation of electromechanical equipment, it is very essential to make measures of accident prevention in order to completely stop similar accidents happening again. To a certain extent, it is meaningful for the crew to be more calm facing to sudden condition.

The software also has a certain scalability. With the continuous development of the technology, there will be a new type of ship machinery parts damage accident. The software maintenance person can add new content, so the software will have more Practicability.

\section{References}

[1] http://msdn.microsoft.com/enus/library/ms723587(VS.85).aspx Microsoft Speech API 5.3

[2] Zou Jian-jun, Accident of ship aircraft damage is analysed (Joural of Shanghai Maritime University), 01 (2003)

[3] Zou Jian-jun, Hu Yi-huai, Human Errors' Analysis in the Shipping Casualties(Joural of Shanghai Maritime University), 24 (2003)

[4] Shao Lian-xin, The Study of Accident of Ship Aircraft Damage of Tianjin Port(Dalian Maritime University), (2009)

[5] Guo Sheng-lu, Zhang Rong-sheng, Visual Basic 6.0(Chinese version) from entry to the master

[6] Liu Jiang, Wang Shi-feng, Xu Shan-lin, Ship Machine Loss Cases Query Software Development based on Microsoft Speech SDK(Mechanical and Electrical Equipment), 28(2011) 